\title{
"Ce n'est pas un livre, mais une pièce de théâtre..." : la lecture de Harry Potter et l'enfant maudit par les utilisateurs du réseau social littéraire Babelio
}

\author{
MARIA EINMAN
}

\begin{abstract}
It's not a book, it's a play...": Harry Potter and the Cursed Child Read by the Users of Babelio, a Social Network for Book Readers. On the $31^{\text {st }}$ of July 2016, Harry Potter and the Cursed Child, a play written by Jack Thorne in collaboration with J. K. Rowling and John Tiffany, was performed for the first time at the Palace Theatre in London. On the same day, the bookshops of Great Britain were literally invaded by the young wizard's fans, whom were curious to know what happens next to their favorite heroes. Thereby, The Cursed Child is a play, not only to be performed, but also - and mostly - to be read. Whereas the Theater Studies, at least in France, consider the drama text mainly as a scenic performance element and thus, limits the drama text reading to text analysis, the reception of Thorne's play allows to ask about the possibility of reading the drama text as a self-sufficient fictional text and invites us to investigate the specificities of this kind of reading. In order to do this, I have analyzed three hundred Cursed Child readers' reviews, which are located on the babelio.com website, one of the biggest French social networks for book readers.

It appears that the Cursed Child's readers tend to read that drama text by comparing it to the seven Harry Potter novels. The main two differences perceived within the scope of this comparison (and which the readers often tend to attribute to the dramatic form specificities) are the so-called lack of descriptions and the rapid reading rhythm. However, these differences may receive a completely opposite evaluation, depending on the reader's openness to the dramatic form. In this way, for the "reluctant" readers, it constitutes an obstacle which prevents the fictional immersion and therefore the pleasure of reading, whereas for the "enthusiastic" readers, it allows them to go through a new and pleasant experience. Moreover, it is not unusual to see that the reading of The Cursed Child markedly reduces the reader's reluctance towards the dramatic form. In fact, the reader reviews analysis proves that The Cursed Child text activates the reader's imagination, regardless of his reluctance. Consequently, the Thorne's drama example demonstrates that a drama text can be considered as a self-sufficient fictional text which is able to arouse the pleasure of reading and we can therefore conclude that the reluctance or the pleasure is led by the reader's own readiness to accept the alterity of the dramatic form.
\end{abstract}

Keywords: Harry Potter and the Cursed Child; drama text; dramatic form; reading; reception; online reader's reviews

DOI: https://doi.org/10.12697/IL.2018.23.1.19 
On est en août 2016. Je me lève de bonne heure pour faire le tour des librairies de Tartu : il faut absolument que je me procure un exemplaire de Harry Potter et l'enfant maudit en version originale que l'on met en vente aujourd'hui. Il le faut absolument, puisque j'ai hâte d'apprendre la suite de l'histoire, et je suis animée par les mêmes sentiments qu'il y a dix ans, en juillet 2007, quelques jours avant la sortie de la dernière partie de la saga : curiosité et impatience. Apparemment, je ne suis pas la seule à les éprouver. Dans la première librairie, on me dit que l'on vient de vendre le dernier exemplaire. Je me précipite dans une autre qui se trouve juste à côté : l'histoire se répète. Enfin, dans une troisième librairie, la chance me sourit : je m'empare du dernier livre qui reste dans le rayon. L'achat réglé, j'entends la caissière dire à une jeune fille que le dernier Harry Potter vient d'être vendu...

En Angleterre, c'est le 31 juillet 2016 que les librairies sont littéralement envahies par les fans de la saga potterienne. Harry Potter et l'enfant maudit, écrit par Jack Thorne en collaboration avec J.K. Rowling et John Tiffany, est publié le jour de sa première représentation au Palace Theatre de Londres. Il s'agit d'une pièce de théâtre ; et quoique selon une idée répandue, une pièce de théâtre ne soit pas pensée pour être lue à l'instar d'un roman, c'est-à-dire essentiellement pour le plaisir de lire, Harry Potter et l'enfant maudit échappe à la règle : si on se le procure, c'est justement pour le lire et pour y prendre plaisir.

\section{Une pièce de théâtre que l'on lit}

Harry Potter et l'enfant maudit ${ }^{1}$ se révèle ainsi être un texte de théâtre qui se distingue résolument des autres textes du même genre sur le plan de la réception. On convient qu'il est rare que des pièces de théâtre se lisent en dehors du cadre scolaire, universitaire ou de la pratique théâtrale ; or L'Enfant maudit, parce qu'elle se voit explicitement reliée à une série de romans célèbres, est une pièce de théâtre que l'on lit de son propre gré, par curiosité ou en cherchant à se replonger dans un monde de fiction familier ; et les lecteurs en sont nombreux. Ainsi, sur le site Babelio, l'un des réseaux sociaux littéraires français, la pièce de théâtre en question compte 4731 lecteurs. À titre de comparaison, même si le nombre de lecteurs de Phèdre ou d'Andromaque de Jean Racine, deux pièces au programme du bac, est plus important (respectivement 8102 et 5421), les statistiques changent considérablement pour les pièces moins connues de l'auteur : le nombre de lecteurs de Britannicus, la troisième pièce de Racine la plus lue sur Babelio, ne s'élève qu'à $3709^{2} \ldots$

Nous l'abrégerons dorénavant, par commodité, en L'Enfant maudit.

2 Pour les pièces contemporaines, les statistiques sont encore plus parlantes : Juste la fin $d u$ monde de Jean-Luc Lagarce, considéré comme un « classique contemporain », 
Le phénomène de L'Enfant maudit nous offre donc l'opportunité de nous intéresser à l'interaction entre le texte dramatique et son lecteur dans le cas où la lecture n'a pas d'autre visée que le plaisir de lire, c'est-à-dire lorsqu'elle n'est pas orientée vers un objectif déterminé par le cadre institutionnel ou professionnel (analyse de texte, mise en scène, etc.) ${ }^{3}$. Sur ce point, notons que le texte dramatique est le plus souvent considéré comme un élément constitutif de la représentation théâtrale : il est ainsi privé de son indépendance. Même au sein des études théâtrales, comme le soulignent judicieusement Jean de Guardia et Marie Parmentier, on a toujours tendance à penser que la lecture du théâtre soit « moins légitime que la représentation $\gg(2009: 131)$. Toutefois, rien n'empêche de lire une pièce de théâtre comme un texte de fiction : la plupart des textes dramatiques comportent tous les éléments nécessaires pour mettre en place l'espace-temps fictionnel dans lequel le lecteur vient s'immerger. Il n'en reste pas moins que ce genre de lecture possède des traits particuliers qui sont mis en lumière par la comparaison avec la lecture d'un texte de fiction narratif. La particularité la plus évidente, mais aussi la plus cruciale, est liée à ce qu'un texte de théâtre ne possède pas d'interface narrative continue. Or en analysant les résultats d'une enquête sur la lecture des textes dramatiques qui a été menée auprès de 120 étudiants en Arts du spectacle, Anick Brillant-Annequin conclut que cette absence peut être perçue par le lecteur comme une ouverture enthousiasmante qui lui permet de laisser libre cours à son imaginaire, mais aussi (et peut-être surtout) comme une « source de malaise » (Brillant-Annequin 2004 : 272-273). Alors que même chez les étudiants en théâtre, prendre plaisir à lire le théâtre ne s'impose pas comme une évidence, qu'en est-il des lecteurs «non spécialistes », en l'occurrence ceux de L'Enfant maudit? En examinant les défis que la lecture de cette pièce leur propose, les appréhensions qu'elle leur inspire et les questions qu'elle soulève chez eux, nous tenterons de comprendre s'il est effectivement possible de prendre plaisir à lire une pièce de théâtre lorsqu'on n'y est pas habitué et de repérer les éléments qui font augmenter ou diminuer le plaisir de lecture ${ }^{4}$.

dénombre 208 lecteurs ; Cendrillon de Joël Pommerat en reste à 110. L'ensemble des données statistiques évoquées dans cet article datent du 26 janvier 2018.

3 Cet article s'inscrit ainsi dans le prolongement de notre thèse de doctorat, dans laquelle nous examinons la question de la lecture des textes de théâtre comme des textes de fiction sous un angle théorique, en nous inspirant notamment du concept de lecteur virtuel de Vincent Jouve.

4 Par le plaisir de lecture, nous entendons le plaisir qu'éprouve le lecteur en refermant le livre : il s'oppose, dans le cas présent, à la déception ou à la frustration. Cette définition est sans doute un peu étroite, mais étant donné la nature de notre corpus d'analyse, elle se révèle pleinement opératoire : via les avis des lecteurs, nous ne pouvons accéder qu'au « résultat final » de la lecture. 
Pour ce faire, nous nous proposons de nous pencher sur les avis des lecteurs de L'Enfant maudit publiés sur le site Babelio (http://babelio.com). Il s'agit de l'un des réseaux sociaux littéraires français les plus importants en France, lancé en 2007 et soutenu par divers organismes publics et institutionnels (Grossetti 2016) ; à ce jour, il compte environ 550000 utilisateurs inscrits. À ceux-ci, Babelio propose de créer leur bibliothèque virtuelle pour y ajouter des lectures passées, en cours ou à venir, mais aussi de les noter sur une échelle allant jusqu'à cinq, ainsi que de publier des avis critiques et d'échanger avec d'autres utilisateurs. Les avis des lecteurs sont accessibles même aux non-inscrits, en proposant donc, il nous semble, un corpus immense pour étudier la réception actuelle des œuvres littéraires, théâtrales, etc. : les travaux qui se servent de ce corpus sont rares $^{5}$, mais cela peut être expliqué par sa constitution relativement récente.

Parmi les 701 avis qui recensent L'Enfant maudit à la date du 18 janvier 2018, nous avons choisi de nous limiter à 300. La première centaine d'avis examinés date de la période qui va du 31 juillet 2016 au 14 octobre 2016, donc de la parution de la version originale de la pièce à la parution de la traduction française du texte : il s'agit d'avis de « grands fans » de l'univers de Rowling qui ont fait le choix de lire le texte en anglais sans attendre qu'il soit traduit ; ce sont aussi les lecteurs les plus enthousiastes et les plus indulgents. La seconde centaine d'avis se rapporte à la période allant du 21 novembre 2016 au 5 janvier 2017 : ces lecteurs font déjà preuve d'une désapprobation plus importante vis-à-vis du texte ; mais on ne s'étonnera pas que les critiques les plus sévères se retrouvent, en général, au sein des avis les plus récents (du 9 juin 2017 au 16 janvier 2018). Il s'avère que dans l'ensemble de ce corpus, environ 70\% des lecteurs accordent de l'importance au fait que L'Enfant maudit est un texte théâtral : c'est à leurs avis que nous allons nous intéresser. Précisons également, afin d'éviter tout malentendu, que même s'il nous arrive d'évoquer des chiffres et des pourcentages, notre ambition n'est pas de fournir ici une étude statistique, le profil social des utilisateurs nous restant inaccessible - nous traitons, métaphoriquement parlant, des motifs qui se rapportent à notre problématique, dans la mesure où les avis de lecteurs, à l'inverse des réponses à une enquête, restent extrêmement hétérogènes.

5 Souvent, ils sont également consacrés à l'étude de la littérature « de jeunesse $\gg$. Voir, à titre d'exemple, Brière-Haquet 2017. 
« Ce n'est pas un livre, mais une pièce de théâtre... »

\section{De la réticence...}

Il va de soi que les lecteurs ne s'intéressent pas à L'Enfant maudit en tant qu'œuvre indépendante : sa réception s'effectue à la lumière des sept parties de la saga, qu'il s'agisse des livres ou des films ; force est toutefois de constater qu'à de très rares exceptions près, on compare la pièce aux romans. Cette comparaison, qui peut également se transposer sur un plan plus général, celui des genres et des formes, détermine magistralement les réactions des lecteurs. Tout d'abord, le passage de la forme romanesque à la forme dramatique, beaucoup moins familière, ne laisse, à lui seul, que rarement indifférent. En général, dans leurs critiques, les lecteurs tendent à souligner d'entrée de jeu que L'Enfant maudit est une pièce de théâtre (sous-entendant que ce n'est pas un roman) ; la plupart d'entre eux établissent par la suite une relation causale entre la forme dramatique et leur expérience de lecture. Dans les avis examinés, la forme dramatique se conçoit comme une sorte de barrière ou d'écran qui s'érige en obstacle entre le lecteur et le plaisir de lecture espéré ; ce dernier est d'ailleurs explicitement relié au désir de « retrouver la magie $\gg$ de l'univers de Rowling, de rencontrer les personnages de la saga et d'apprendre la suite de l'histoire, mais très souvent, ce désir, et donc le plaisir de lecture, se voit contrarié par le caractère inhabituel de la forme. L'opacité de l'écran de la forme dramatique varie selon les lecteurs, dont les réactions se répartissent ainsi entre deux pôles : la réticence extrême à la forme et l'acceptation enthousiasmée de celle-ci ; mais les cas absolus sont bien évidemment rares : la plupart des réactions sont moins radicales, teintées d'appréhension, de curiosité, voire d'indifférence. Précisons donc d'emblée que s'il nous arrive d'évoquer les « lecteurs réticents » ou les « lecteurs enthousiastes », il s'agit rarement des lecteurs parfaitement réticents ou enthousiastes vis-à-vis de la forme dramatique ou de l'œuvre en général ; pour la plupart, les critiques de L'Enfant maudit s'avèrent mitigées ; la réticence et l'enthousiasme, sauf mention contraire de notre part, ne sont ainsi jamais absolus.

Nous allons d'abord nous intéresser aux tenants et aboutissants de la réticence à la forme dramatique qui influe, de manière non négligeable, sur l'immersion du lecteur dans la fiction ${ }^{6}$. Cette réticence peut se révéler plus ou moins importante : soit les lecteurs expliquent leur déception principalement par ce qu'ils croient être le propre de la forme dramatique - en d'autres termes, pour eux, la forme « gâche tout $\gg$ ou peu s'en faut ; soit ils avouent simplement ne pas aimer ou ne pas avoir l'habitude de lire le théâtre. Parmi les raisons de la réticence à la forme, on repère,

6 Par immersion dans la fiction, nous entendons ici, avec Jean-Marie Schaeffer, l'état dynamique du lecteur qui « vient habiter » une œuvre fictionnelle tandis que le monde réel reste à la lisière de sa perception (Schaeffer 1999 : 182). 
entre autres, la difficulté de « s'accrocher » à l'histoire et la nécessité de fournir un effort plus important pour pouvoir rentrer au sein du monde fictionnel, la forme dramatique ne favorisant pas ainsi l'immersion :

Ceci dit, il ne s'agit pas d'un roman mais du script d'une pièce de théâtre [...] Par conséquent, il est forcément plus difficile de rentrer dans l'histoire et de s'attacher aux nouveaux personnages... (Thecosmicscam, 28/11/2016 $)$

La difficulté susdite semble, quant à elle, découler d'une première particularité de la pièce de théâtre en question qui surgit essentiellement de la comparaison de L'Enfant maudit aux romans de Rowling : on déplore notamment l'absence ou le manque de détails et/ou de descriptions des décors et des personnages. Ce manque rebute la plupart des lecteurs réticents et traverse comme un fil rouge l'ensemble des critiques ; il faut noter cependant que les didascalies de la pièce tendent plutôt à le pallier. Elles rendent compte non seulement des objets qui se trouvent dans l'espace scénique et des actions physiques qui y ont lieu, mais aussi des intentions, de l'état émotionnel et des pensées des personnages ${ }^{8}$; toutefois, la richesse relative des didascalies ne satisfait pas les lecteurs réticents, chez qui le manque de détails fait souvent naître un sentiment de frustration face au texte. Ils sentent que ce manque ne leur permet pas de retrouver l'ambiance et la « profondeur » souhaitées ; il en va de même pour les caractères des personnages qu'ils traitent souvent de superficiels. Le terme de profondeur revient à maintes reprises dans les avis examinés ; on attribue ainsi, par la négation, une certaine platitude au texte de la pièce :

En effet, vous n'avez pas de descriptions $[\ldots]$ On ne va de ce fait pas au fond des choses et ça manque évidemment de profondeur. (VibrationLittéraire, 20/08/2016)

$[\mathrm{L}] \mathrm{e}$ côté pièce de théâtre ne permet guère d'approfondir la psychologie des personnages. Nous restons en surface. (prunelle337, 05/12/2016)

Pour référencer un avis, nous donnons entre parenthèses le nom d'utilisateur et la date de publication : ces deux informations doivent suffire pour le repérer rapidement depuis la page du site Babelio regroupant les avis de lecteurs (en l'occurrence https:// www.babelio.com/livres/Thorne-Harry-Potter-et-lEnfant-Maudit/860109/ critiques?tri=dt). L'orthographe et le style des avis n'ont pas été modifiés.

8 Pour n'en donner qu'un exemple, voici la didascalie initiale de la scène 19 du troisième acte : « Poudlard, sur le terrain de quidditch. La scène s'ouvre sur Delphi qui se délecte de sa nouvelle identité. Là où on sentait un malaise, une insécurité, on ne perçoit plus que la force du pouvoir $\gg$ (Rowling, Tiffany, Thorne 2016). 
Un autre aspect qui agit sur l'immersion et qui peut faire croître la réticence du lecteur est le changement du rythme de la lecture : dans ce cas de figure, celuici est notamment perçu comme étant excessivement haché ou bien, ce qui est plus fréquent, trop rapide. D'une part, cette perception dépend, une fois de plus, du passage de la forme romanesque à la forme dramatique : L'Enfant maudit est évidemment plus court et se lit plus rapidement que n'importe lequel des sept romans. D'autre part, cette perception, semble-t-il, est due à la nature de la pièce à proprement parler. Si nous cherchons à définir le genre de L'Enfant maudit, nous pouvons postuler que cette pièce se rapproche d'un mélodrame (qu'il faut ici entendre hors de toutes les connotations négatives du terme) : outre le fait qu'elle se fonde sur un conflit familial, tout à fait typique de ce genre, et qu'elle met en œuvre un dénouement où, pour ainsi dire, la vertu triomphe et le vice est puni, et qu'elle se sert d'un secret, ou plutôt d'un mystère, comme moteur dramaturgique, L'Enfant maudit se caractérise par un enchaînement rapide de péripéties, censé piquer sans relâche la curiosité du spectateur - ou du lecteur, tout cela sans évoquer la dimension spectaculaire, c'est-à-dire la présence de nombreux « clous visuels ». De plus, comme le fait remarquer Florence Fix, le mélodrame se fonde sur la « juxtaposition continue de micro-séquences »-que l'on retrouve bel et bien dans la pièce de Thorne -, ce qui permet « de donner à l'ensemble un rythme fébrile, de créer une série de situations-limites dans la surenchère permanente de l'émotion et de l'attente $\gg(2011: 151)$.

De ce point de vue, L'Enfant maudit devrait donc littéralement absorber l'attention du lecteur tout en favorisant l'immersion dans la fiction. Toutefois, les lecteurs réticents considèrent le rythme rapide de la lecture de manière plutôt négative, en le reliant toujours au manque de profondeur :

Je suis quand même frustrée car il s'agit d'une pièce de théâtre [...] c'est rapide, furtif, et juste une histoire « en surface ». (nannou71, 4/12/2016)

Une pièce de théâtre est assez froide et ne retrace pas forcément toute l'ambiance de l'univers Harry Potter [...] tout va trop vite, et à peine commencé, nous voici déjà à la dernière scène après quelques heures de lecture $[\ldots]$ je ressors de ce livre presque frustrée. (Hanta, 25/11/2016)

Ainsi, le manque de descriptions (par rapport aux romans) et un rythme de lecture singulier apparaissent comme deux éléments majeurs qui constituent, dans le contexte de la réception de L'Enfant maudit, ce que nous avons choisi d'appeler l'écran de la forme dramatique : pour une partie des lecteurs, cet obstacle s'avère insurmontable. Dès lors, l'immersion dans la fiction devient complexe - ce n'est pas par hasard, nous semble-t-il, que les remarques sur le caractère superficiel du texte sont si nombreuses -, et fait souvent naître une sorte de frustration ou de déception. 


\section{... à l'acceptation}

On repère aussi des traces de réticence dans les avis des lecteurs qui ont généralement apprécié la pièce ; or chez ces derniers, nous pouvons observer une stratégie de réception différente : celle-ci implique l'ouverture à l'altérité de la forme et se décline, en général, selon trois modalités. D’abord, il y a des lecteurs, relativement peu nombreux, qui tiennent à préciser qu'ils aiment lire le théâtre : même si certains d'entre eux font remarquer que la forme dramatique « ôte une certaine profondeur aux personnages » (SuzyBess, 24/11/2016), la plupart de ces lecteurs trouvent la lecture prenante ; et, en plus de considérer L'Enfant maudit comme une « expérience tout à fait inédite 》 (ManonsBooks, 4/01/2017) justement grâce à sa forme, ils indiquent aux lecteurs « néophytes » des pistes pour rendre la lecture plus amusante, voire plus plaisante, notamment en mettant en œuvre la polyphonie propre au texte dramatique :

Mais si j'ai un conseil à vous donner c'est de le lire à plusieurs. Retrouvez-vous entre amis [...] Répartissez-vous les personnages présents et éclatez-vous ! [...] Si vous vous prenez au jeu et que vous allez jusqu'à faire des intonations particulières [...] le moment n'en sera que meilleur. (LesLecturesdOriane, 14/09/2017)

Un certain nombre de lecteurs constatent simplement que la forme « ne les a pas gênés » : la lecture, dans ce cas, s'avère une expérience plutôt agréable. Quoiqu'ils évoquent toujours l'absence de détails et le rythme de lecture excessivement rapide, tout en regrettant de ne pas avoir « une vraie suite narrative $\gg$ (clairebossier, 15/01/2018), ces lecteurs arrivent à se rendre compte qu'un texte de théâtre ne doit pas fonctionner de la même manière qu'un roman : «Vu que ce n'est pas un roman, le mode de narration n'est pas le même. Cela ne m'a pas dérangée plus que ça ! », s'exclame l'utilisateur PeaceandBooks (19/12/2016). «[C]'est une pièce de théâtre qui respecte les codes du genre. Pas de déception pour moi $\gg$, conclut l'utilisateur Emiliec28 (2/10/2016). Nous pouvons en outre constater que chez ce groupe de lecteurs, le désir de se replonger dans un monde fictionnel familier l'emporte sur la réticence quant aux « défauts » attribués à la forme, et participe donc à l'ouverture à l'altérité. Ainsi, l'utilisateur Blackbird, lorsqu'il observe que le passage du roman au théâtre ne l'a pas perturbé, ajoute pour justifier son propos : « Pourvu que J.K. Rowling continue de nous transporter dans cet enchantement qu'est l'univers d'Harry Potter $\gg(5 / 01 / 2017)$.

Enfin, l'échantillon le plus intéressant, car il témoigne, sur un certain plan, de la capacité de L'Enfant maudit à faire oublier à son lecteur les préjugés qu'il pouvait avoir sur les textes de théâtre, comprend des lecteurs dont la réticence 
«e n'est pas un livre, mais une pièce de théâtre... »

première, se traduisant par une sorte d'appréhension, d'effroi ou de déstabilisation face à la forme dramatique, se mue en acceptation garante du plaisir de lecture. Dans ce cas-ci, l'agencement mélodramatique de l'intrigue et un rythme de lecture plus intense, dépréciés par des lecteurs réticents, sont le plus souvent évalués de manière positive (comme c'est par ailleurs le cas des deux premiers groupes de lecteurs évoqués), car ces aspects, comme nous l'avons dit, permettent une immersion rapide au sein du monde fictionnel. Ainsi, les lecteurs sont souvent amenés à conclure, parfois non sans étonnement, que la forme ne change rien au caractère captivant de la fiction :

Je vous avouerais que j'étais un peu réticente du format pièce de théâtre, j'avais peur de ne pas être en immersion totale dans l'histoire. Mais heureusement, on oublie vite l'apparat du script, il se passe tellement de chose qu'on est pris par l'action! (LeaneBelaqua, 11/08/2016)

[J]'avais tort d'avoir autant d'a priori [...] Malgré le fait qu'il s'agit d'un script, je n'ai eu aucun problème pour m'immerger dans cette histoire que j'ai trouvé fantastique. Bien sûr, ça n’a pas la force d'un roman [...] Mais étrangement, ce n'est pas gênant et ça n'empêche en rien l'imagination de carburer. (Saefiel, 31/07/2016)

Précisons, pour rester objective, qu'à côté des lecteurs « convertis », on trouve bien évidemment ceux dont l'appréhension ou la déstabilisation face à la forme ne se transforme pas en une réaction positive, mais en même temps, un certain nombre de lecteurs qui disent ne pas du tout apprécier la lecture des pièces de théâtre reconnaissent que L'Enfant maudit se lit rapidement et agréablement. «On se laisse facilement séduire [... n'avoir que très peu d'éléments descriptifs fait carburer l'imagination ! », s'enthousiasme l'utilisateur unpointcesttout qui exprime, quelques lignes plus haut, sa réticence « à l'idée de lire une pièce de théâtre $\gg(9 / 09 / 2016)$. Il est à noter d'ailleurs que pour ce lecteur, le manque de descriptions se transforme en un aspect positif dans la mesure où il est susceptible de mettre en branle l'imagination ; et de manière plus générale, on peut dire que les lecteurs pour lesquels la forme dramatique ne constitue pas ou plus d'écran imperméable, mettent également en valeur la manière dont la pièce de Thorne active leur imaginaire.

\section{Deux manières d'imaginer}

On peut convenir sur ce point, avec Anick Brillant-Annequin (2004 : 271-272), que le texte de théâtre offre une liberté plus importante à l'imagination du lecteur, 
en raison justement d'absence d'interface narrative continue. Selon les résultats de son enquête que nous avons déjà évoquée, pour la plupart des répondants, la lecture du théâtre se définit « par des termes relevant du champ de l'imaginaire »; et lorsqu'un tiers de lecteurs valorisent « le libre-champ laissé à l'imaginaire », $21 \%$ de «non-lecteurs » (c'est-à-dire des répondants qui lisent peu de façon générale) le critiquent (ibid.). En d'autres termes, cet aspect propre à la lecture d'un texte théâtral est susceptible d'être évalué de manière opposée en fonction de l'expérience du lecteur ; et une ambiguïté du même ordre se présente dans les avis des lecteurs de L'Enfant maudit.

En ce qui concerne les lecteurs réticents, d'aucuns font remarquer que le manque de descriptions et la prépondérance des dialogues les empêchent de visualiser les scènes ; tandis que d'autres mettent en lumière l'effort que nécessite l'imagination : « Il m'a fallu me concentrer pour imaginer les décors et les effets spéciaux $\gg$, s'indigne l'utilisateur dlcb26, profondément perturbé par le « format pièce de théâtre $\gg(30 / 08 / 2017)$. « Ici il faut faire un effort d'imagination et c'est plus compliqué pour savourer l'histoire... », observe l'utilisateur mara qui, selon ses dires, n'est pas habituée à lire les pièces de théâtre (24/08/2016). En même temps, on ne s'étonne pas que les opinions des lecteurs « enthousiastes » soient d'une nature différente : comme nous l'avons constaté, même s'ils peuvent toujours regretter le manque de descriptions, celui-ci ne semble pas nuire au processus de la lecture. Ces lecteurs peuvent valoriser la possibilité de concevoir leur propre mise en scène imaginaire à partir du texte, souligner que l'absence de descriptions ne les gêne pas, puisque les dialogues suffisent pour imaginer les personnages, et confirmer que les didascalies sont assez détaillées pour visualiser l'espace-temps fictionnel. Certains lecteurs font de même remarquer que si on a lu les romans, on ne devrait avoir aucune difficulté à se représenter les lieux et les décors familiers ; ainsi, dans leurs avis, les romans et la pièce de théâtre, au lieu de s'opposer, se complètent :

Étant donné que nous connaissons le physique des personnages par cœur, ainsi que la représentation des lieux, le fait que rien ne soit décrit dans le livre n'est pas vraiment pénalisant. (LadyChantilly, 16/09/2017)

Outre les critiques relativement peu nombreuses qui témoignent explicitement de l'activation de l'imagination par le texte de la pièce, nous pouvons repérer dans l'ensemble des impressions un leitmotiv qui nous semble également confirmer, de manière implicite, le pouvoir imageant de L'Enfant maudit. Très souvent, les lecteurs, qu'ils soient plutôt réticents ou plutôt enthousiastes, notent que la lecture de la pièce leur a donné l'envie de voir le spectacle : non seulement parce qu'ils 
« Ce n'est pas un livre, mais une pièce de théâtre... »

sont enclins à penser qu'un texte de théâtre en général ou la pièce de Thorne en particulier ne valent que par la réalisation scénique ${ }^{9}$, mais aussi parce qu'ils imaginent que ce spectacle devrait être « fantastique », « spectaculaire » et « impressionnant » - or c'est la lecture du texte (et plus précisément celle des didascalies) et donc, on peut le supposer, les images mentales qu'elle fait surgir qui doit les inciter à le penser ${ }^{10}$.

Cela dit, l'envie de voir L'Enfant maudit en spectacle se manifeste aussi lorsque le lecteur essaie de s'imaginer la mise en scène effective de la pièce d'après les indications textuelles : il n'est alors pas rare qu'il termine par conclure, en partant de son expérience de spectateur ou de ses connaissances sur le théâtre, qu'il aimerait bien voir le spectacle, parce que ce dernier lui semble quasiment irréalisable. $\ll[C ̧]$ a donne super (super) fort envie de voir la pièce qui doit être tout simplement grandiose ! » s'exclame l'utilisateur bykiss, qui précise : «à plusieurs reprises, on se demande ce que telle situation pourrait donner sur une scène de théâtre, comment ils ont pu rendre tel retournement sans les effets spéciaux des films, etc. » (4/10/2016); «j'ai d'ailleurs très envie de la voir [la pièce] pour savoir comment les "effets" auront été créés », lui fait écho l'utilisateur SapereAude (20/09/2016). Ainsi, l'imagination du lecteur de L'Enfant maudit, en plus d'être sollicitée par la dimension fictionnelle du texte, l'est également par sa dimension scénique qui va de pair avec l'idée de la représentation effective : cela vient confirmer le propos de Guardia et Parmentier selon lequel la spécificité de la lecture des textes dramatiques, sur le plan de l'imaginaire, « est de se mouvoir dans un étrange entre-deux : représentation au premier degré (d'une fiction), représentation au second degré (d'une représentation physique d'une fiction) » (2009 : 145). Or l'importance de la composante scénique dans l'imaginaire du lecteur peut être renforcée, semble-t-il, par la présence de la création effective de ce texte dans le champ médiatique. Les lecteurs de L'Enfant maudit veulent voir la pièce en spectacle et tentent de s'imaginer sa mise en scène parce qu'ils se rendent compte, entre autres, que le spectacle est joué actuellement : ainsi, dans les avis qui suivent la parution du texte anglais de la pièce (et donc la première du spectacle), ce désir est nettement plus explicite et plus intense que dans les critiques plus tardives, car la mise en scène de la pièce n'est plus aussi intensément rapportée par les médias. Mais quoi qu'il en soit de la composante scénique de l'imaginaire, nous pouvons constater que de manière générale, moins le lecteur de L'Enfant

9 Cf. « Après c'est du théâtre donc le théâtre c'est pour que ce soit vu et non lu » (dixdoudou, 13/09/2016) ; « ... mais je fais tout de même partie de ceux qui pensent qu'une pièce de théâtre ça se joue, ça ne se lit pas ! (Blondreader, 26/12/2016), etc.

10 Cf. « ... les détails décrits dans chaque scène font qu'on s'imagine très bien la pièce. Tellement que j'ai bien envie de la voir en vrai. » (Luria, 22/08/2016) 
EINMAN

maudit est réticent à la forme dramatique, plus son imagination est activement sollicitée par le texte, ce qui soutient indéniablement l'immersion dans la fiction, et donc le plaisir de lecture.

\section{Laltérité de la forme}

L'interrogation sur le rapport entre le texte de L'Enfant maudit et sa réalisation scénique, outre qu'elle permet de nuancer le fonctionnement de l'imagination propre à la lecture d'un texte de théâtre, met en lumière un autre phénomène curieux : si certains lecteurs doutent de la « faisabilité » de la mise en scène, c'est que leur représentation générale du théâtre se fonde sur des idées reçues qui peuvent altérer l'expérience de lecture. Cette interrogation surgit, par exemple, lorsque le lecteur est convaincu que la seule fonction des didascalies est de décrire l'espace scénique et que l'équivalence entre celui-ci et celles-là doit être parfaite, ce qui peut l'amener à accuser L'Enfant maudit d'une sorte d'invraisemblance, en allant jusqu'à lui refuser le droit de se nommer une pièce de théâtre. Ainsi, d'une part, les didascalies ne devraient pas rendre compte des sentiments et des pensées des personnages : « [V] ous ne m'aurez pas, ce texte n'est pas une vraie pièce de théâtre », déclare l'utilisateur Kimysmile, « voir autant d'informations dans les didascalies m'a fait grincer les dents à plusieurs reprises $\gg(27 / 12 / 2016)$. D'autre part, le changement constant des temps et des lieux que les didascalies postulent est aussi considéré comme littéralement impossible à reproduire sur scène, contrevenant aux règles de la composition dramatique :

L'Enfant maudit est censée être une pièce de théâtre, mais me semble a priori injouable, à moins de subir de sérieux remaniements. Les didascalies sont complètement irréalistes [...] L’unité de temps et de lieu vole en éclat [...] Résultat, on a surtout l'impression que le format théâtral a été choisi par paresse intellectuelle. (Arakasi, 9/08/2017)

On voit donc que si la pièce de théâtre ne coïncide pas avec l'image que le lecteur s'est faite des pièces de théâtre en général (donc, d'une certaine manière, avec son horizon d'attente), il éprouve une sorte de frustration - de même qu'un lecteur qui doit faire un effort pour imaginer l'espace-temps fictionnel ou qui ne retrouve pas dans L'Enfant maudit les descriptions détaillées et la « profondeur » des romans de Rowling. Dans ce dernier cas, d'ailleurs, il est également probable que la réception soit partiellement influencée par des idées reçues ${ }^{11}$. Cela dit,

${ }_{11}$ Bien sûr, rien n'empêche d'imaginer un lecteur pour qui L'Enfant maudit est sa première lecture «théâtrale » : dans ce cas, il n'est pas exclu que son avis sur les textes de théâtre 
«e n'est pas un livre, mais une pièce de théâtre... »

les lecteurs peuvent être convaincus que c'est la forme dramatique en tant que telle qui ôte de la « profondeur » au texte et aux personnages ou bien encore que la forme dramatique ne permet pas de « développer » l'intrigue (d'où le changement du rythme de lecture) :

L'histoire aurait pu être intéressante s'ils avaient choisi un meilleur format, un support « roman » aurait pu permettre un meilleur développement de l'histoire. (CBreval, 4/11/2017)

$[\mathrm{C}]$ e n'est pas un livre mais une pièce de théâtre. [...] Très orienté dialogue, la narration fait fi des pensées, de la psychologie et de l'action. (quoilire, $16 / 09 / 2016)$

Sans aller à la quête de la source de ces a priori qui contribuent à augmenter la réticence du lecteur, notons simplement que la tendance à faire des généralisations tout en transformant en défauts les particularités de la forme dramatique nous semble être en rapport avec l'ouverture d'esprit du lecteur et la conscience qu'il prend à l'égard de l'œuvre ; les lecteurs que la forme dramatique ne gêne pas, voire qu'elle enthousiasme, sont également le plus à même de (se) rendre compte de son caractère spécifique sans pour autant oublier qu'il s'agit d'une œuvre de fiction. Qui plus est, l'analyse des avis des lecteurs nous permet de conclure que dans le cas de L'Enfant maudit, le manque de descriptions, l'accélération du rythme de lecture et une manière spéciale d'activer l'imagination peuvent recevoir des évaluations complètement opposées : cela incite à penser qu'en eux-mêmes, ces aspects sont neutres, et que leur évaluation dépend surtout de l'aptitude du lecteur à accepter l'altérité de la forme dramatique. Si le lecteur prend la forme dramatique pour ce qu'elle est, elle ne gênera en rien la lecture ni le plaisir que l'on peut en tirer $^{12}$; en revanche, s'il essaie de la faire rentrer, coûte que coûte, dans le cadre de son système de représentations, il risque de fermer le livre frustré et déçu.

Pour simpliste que cette conclusion puisse paraître, elle n'en reste pas moins vraie ; de plus, nous semble-t-il, elle s'applique à la lecture de manière générale, concernant non seulement la forme, mais aussi le fond d'une œuvre. Et pourtant, même s'il semble aller de soi que la lecture des œuvres de fiction est un exercice

en général soit influencé principalement par la comparaison qu'il établira entre les romans de Rowling et la pièce.

12 Cela dit, une fois que le lecteur a accepté la forme, il lui reste encore à accepter le fond faisons simplement remarquer que même lorsque les lecteurs de L'Enfant maudit ne sont pas perturbés par le « format pièce de théâtre », ils peuvent reprocher à cette pièce l'incohérence des détails, le manque de vraisemblance sur le plan de l'intrigue, etc. La forme dramatique ne constitue qu'un obstacle parmi d'autres, mais c'est aussi le premier obstacle auquel le lecteur vient se heurter et qui est souvent le plus difficile à franchir. 
d'empathie, on ne souligne que rarement que l'empathie, qui consiste justement dans le fait d'accepter l'altérité, nécessite un effort mental et même éthique, dans la vie comme dans la lecture, car elle demande au sujet de mettre à l'écart son moi. Selon Maurice Blanchot, la lecture est menacée surtout par l'acharnement du lecteur « à vouloir demeurer lui-même en face de ce qu'il lit, à vouloir être un homme qui sait lire en général $\gg(1955$ : 263) : dès que le lecteur se heurte à quelque chose qui contredit les idées qu'il s'est faites sur la lecture, celle-ci, pour ainsi dire, ne fonctionne plus, le privant d'immersion et donc de plaisir les exemples cités plus haut en attestent ; pour que la lecture « marche bien $\gg$, il lui faut tâcher d'accepter le texte. C'est aussi dans ce sens que le texte, pour fonctionner, demande une coopération de la part du lecteur. Il semble d'ailleurs que L'Enfant maudit, dans la mesure où chez ses lecteurs, la curiosité d'apprendre la suite de l'histoire de Harry Potter prime souvent sur l'appréhension ou la réticence envers le format inhabituel, peut favoriser l'effort d'acceptation ou d'ouverture, et même le récompenser, lorsque le lecteur découvre que cette pièce de théâtre se lit tout aussi agréablement qu'un Potter en lui offrant, de plus, une expérience tout à fait nouvelle - celle de l'altérité.

\author{
Maria Einman \\ maria.einman@gmail.com \\ Kaunase pst 26-1 \\ 50706 Tartu \\ EESTI / ESTONIA
}

\title{
Bibliographie
}

Avis des lecteurs de Harry Potter et l'enfant maudit : https://www.babelio.com/livres/ Thorne-Harry-Potter-et-lEnfant-Maudit/860109/critiques?tri=dt (26.01.2018).

Blanchot, M. 1955. L'Espace littéraire. Paris : Gallimard.

Brière-Haquet, A. 2017. Twilight : une critique en clair-obscur. - Strenae, 12, https:// journals.openedition.org/strenae/1730 (12.02.2018).

Brillant-Annequin, A. 2004. Lire des pièces de théâtre - le pari de l'impossible ? - Langlade G., Rouxel A., dir., Le Sujet lecteur. Lecture subjective et enseignement de la littérature.

Rennes : Presses Universitaires de Rennes, 267-278.

Fix, F. 2011. Le Mélodrame : la tentation des larmes. Paris : Klincksiek.

Grossetti, L. 2016. Les réseaux sociaux littéraires : les exemples de Booknode et Babelio. - http://mondedulivre.hypotheses.org/5224 (12.02.2018).

Guardia, J. (de), Parmentier, M. 2009. Les yeux du théâtre. Pour une théorie de la lecture du texte. - Poétique, 158, 131-147.

Rowling, J.K., Thorne, J., Tiffany, J. 2016. Harry Potter et l'enfant maudit. Paris : Gallimard. Schaeffer, J.-M. 1999. Pourquoi la fiction? Paris : Seuil. 